\title{
Auto-stratification in drying colloidal dispersions: Experimental Investigations
}

\author{
R. E. Trueman ${ }^{1}$, E. Lago Domingues ${ }^{2}$, S. N. Emmett ${ }^{2}$, \\ M. W. Murray ${ }^{2}$, J. L. Keddie ${ }^{3}$ and A. F. Routh $* 1$ \\ ${ }^{1}$ Department of Chemical Engineering and Biotechnology, \\ University of Cambridge, Pembroke Street, Cambridge CB2 3RA, UK \\ ${ }^{2}$ AkzoNobel, Wexham Road, Slough SL2 5DS, UK \\ ${ }^{3}$ Department of Physics, University of Surrey, Guildford GU2 7XH UK \\ Corresponding author: afr10@cam.ac.uk
}

\begin{abstract}
In films cast from a colloidal dispersion comprised of two particle sizes, we experimentally examine the distribution of particles normal to the substrate. The particle concentrations at various positions in the film are determined through atomic force microscopy and NMR profiling. The results are compared to a previously derived diffusional model. Evidence for diffusional driven stratification is found, but the importance of other flows is also highlighted. The conditions that enhance particle stratification are found to be a colloidally stable dispersion, low initial volume fractions, a low concentration of the stratifying particle and for the Peclet numbers of the two components to straddle unity.
\end{abstract}




\section{Introduction}

Drying of latex particle blends can lead to non-uniform particle distributions in the direction normal to the substrate $[1,2]$. Control over the stratification process could lead to multi-functional coatings. Alternatively there are applications when a uniform particle distribution is required. For example even distribution of opacifier is required in films to ensure uniform opacity. To create a latex film the wet dispersion is first applied to a substrate. Evaporation of solvent brings the particles into close packing, and ideally particle deformation then occurs to create a structure without voids. Finally diffusion of polymer chains across particle boundaries imparts mechanical strength and results in the final homogeneous film [3]. The initial drying step allows non-uniform particle distributions to be created and this is the subject of this work.

Drying of dispersions from an initial wet state to a form of close packing has been studied extensively. A common observation is of non-uniform drying with fronts of close packed particles passing laterally across films [4]. The reason for this is packing of particles in regions of lower height, followed by continued evaporation from these regions after particles have been consolidated. The lateral drying has been termed the coffee ring effect, after the original work by Deegan et al. [5]. The lateral drying also results in pressure gradients and film cracking [6, 7].

Vertical stratification is seen in films of sufficient lateral dimension, so that horizontal drying is not dominant. There is a competition between timescales to determine if the well known particle accumulation at the film surface is observed. The top surface of the film is descending at a rate governed by evaporation. The particles that are collected by this descending front can either diffuse away, or, if they are too slow moving, they gather into a descending "piston". For a film of initial thickness $H$ evaporating at a rate $\dot{E}$, the balance between the particle diffusion and the applied convection is captured in the Peclet number, $P e$.

$$
P e=\frac{6 \pi \eta R H \dot{E}}{k T}
$$


where $\eta$ is the solvent viscosity, $R$ the particle radius and $k T$ thermal energy. For $P e>>1$, particle diffusion is irrelevant and a non-uniform particle distribution is seen during drying. For $P e<<1$, diffusion is dominant and a uniform distribution is observed. A diffusional model for single component dispersions was previously published by Routh and Zimmerman [8] and a scaling prediction that the particle concentration is proportional to $P e^{1 / 2}$ was broadly in agreement with experimental measurements [9].

\subsection{Review of Diffusional Model}

Modelling of auto-stratification in drying latex films was carried out by Trueman et al. [10]. In their model, a film is composed of two types of particles dispersed in a continuous solvent. Evaporation from the top surface reduces the film height and the particles distribute themselves according to standard diffusional laws. The volume fraction evolution is predicted by writing the diffusional flux of each component as being driven by the gradient in chemical potential. As dispersions become more concentrated, the motion of particles become hindered. This effect is captured within a sedimentation coefficient. The chemical potentials of the different components (two particle species and the solvent) are linked through the Gibbs-Duhem equation [11]. The chemical potential of the solvent is related to the osmotic pressure, which diverges as the particles come into close packing. The functional form of this divergence is contained within the compressibility. In principle, both the compressibility and the sedimentation coefficient are measurable quantities. The ratio in the chemical potential gradients for the two particle species is the final piece of information needed to provide solvable equations. In principle, any chemical potentials are possible with the surface chemistry of the particles being crucial. For a first set of solutions [10], it was assumed that the particles are non-interacting, with chemical potentials merely determined by entropy. A follow-up piece of work [12] has shown how particle interactions affect the final particle distributions in films. An implicit assumption in the model is that the dispersion remains colloidally stable throughout the concentration range.

The derived equations for predicting auto-stratification [10] are complex. As will become ev- 
ident, we do not know the interaction potential between different particle types in the present experiments. Furthermore the experimental techniques cannot provide information during the drying process but are applied only to fully dry films. Quantitative measurements of concentration of two particle types as a function of position are challenging to obtain in experiments. Hence a quantitative test of the theory is not feasible and the aim of this paper is to examine experimentally whether stratification in multi-component, colloidal drying films is possible and whether the derived model is useful for a qualitative understanding.

The model predicted that maximum stratification would be observed in dilute dispersions when the Peclet numbers of the two particle types straddled unity. This meant $P e_{2}$ being larger than unity and $P e_{1}$ being below unity with $P e_{1} P e_{2} \approx 1$. Larger particles (with $\mathrm{Pe}>1$ ) will accumulate at the top of the film, whereas there will be a higher concentration of small particles (with $\mathrm{Pe}<$ 1) near the bottom. In addition as large a difference in Peclet numbers as possible increases the concentration gradient of particles. The easiest way to achieve different Peclet numbers in a two component film is to use differing particle sizes, such that $\mathrm{Pe}>1$ for one component and $\mathrm{Pe}<1$ for the other.

In a blend of colloidal particles with two different particle sizes, $\dot{E}, T$, and $\eta$ are the same for both populations of particle. The ratio in Peclet number for the large and small particles, $P e_{1} / P e_{2}$, is set by the ratio of the particle radii, $R_{1} / R_{2}$. As the evaporation rate of a drying colloidal film is raised or lowered, then the geometric mean, $\sqrt{P e_{1} P e_{2}}$ is raised or lowered in proportion. In our experiments, the ratio of the particle radii and the evaporation rate are both systematically and independently adjusted as a means to vary $P e_{1} / P e_{2}$ and $\sqrt{P e_{1} P e_{2}}$. 


\section{Materials and Methods}

\subsection{Latex samples}

Acrylic latices with a range of sizes were made by emulsion polymerization using a persulphate initiator. This ensured that the particles all have a similar surface chemistry. The polymer had a relatively high glass transition temperature (Tg) to minimise artefacts and damage during AFM imaging and to prevent the acquisition of an NMR signal at room temperature, hence making the polymer "invisible" in these experiments. A separate latex was required for imaging in NMR experiments and it is required to have very high polymer mobility. After experimentation with a number of samples it was determined that a poly(isodecyl acrylate) colloidal latex synthesized by M. Rabjohns in the laboratory of Prof. Peter Lovell at the University of Manchester was the most suitable, considering its particle size and observed signal strength. This latex was produced using a seeded semi-batch emulsion polymerization method. The specifications of all the particles can be seen in Table 1. Dynamic Light Scattering (DLS) using a Brookhaven ZetaPALS was employed to find the average particle size. The glass transition temperature, was measured using differential scanning calorimetry (DSC).

Table 1: Characteristics of latex samples.

\begin{tabular}{c|c|c|c|c} 
Sample code & DLS Diameter $(\mathrm{nm})$ & AFM Diameter $(\mathrm{nm})$ & $T_{g}\left({ }^{\circ} \mathrm{C}\right)$ & Solids wt \% \\
\hline \hline Acrylic 1 & 130.1 & 109 & 69 & 53.6 \\
Acrylic 2 & 224.6 & 208 & 68 & 52.4 \\
Acrylic 3 & 381.8 & 367 & 69 & 54.2 \\
Acrylic 4 & 544.7 & 528 & 69 & 53.6 \\
Acrylic 5 & 645.9 & 557 & 70 & 53.9 \\
poly(isodecyl acrylate) & 172.7 & N/A & -50 & 54.2
\end{tabular}

\subsection{Film casting and drying}

Blended samples were made from a combination of two samples of latex dispersion and deionised water to produce the required particle volume fraction for each component. Samples were blended 
in glass vials ranging from $2.5 \mathrm{~mL}$ to $25 \mathrm{~mL}$ in size, depending on the amount required. The dispersions and water were added via pipette, with the water being added before the dispersions. Adequate mixing of the blends was ensured through use of a Topmix FB15024 vibrating plate. The blends were then pipetted onto glass slides, with the pipette tip being used to spread the dispersion. It was required to dry the films at varying evaporation rates in order to change the Peclet numbers. Whilst it is possible to speed up the drying process by blowing air across the top of films $[13,14]$, for most experiments it was necessary to dry the films at a slower rate than obtainable under ambient conditions. To facilitate this the films were placed within a chamber which had holes that had been drilled into the top. These holes were of varying diameter in order to alter the drying rate. A petri dish of water was also placed in this chamber. This increased the humidity up to a steady state more quickly, and also facilitated measurement of the evaporation rate (in $\mathrm{m} / \mathrm{s}$ ) from the water mass loss from the petri-dish. This evaporation rate was then taken to be the same as that of the drying film.

A perennial problem was edge drying. This was minimised by applying a plastic laminate coating onto the glass slide substrates. A hole cut into the center formed a walled container that, when a film is applied, the thickness at the edges does not reduce to zero, thus reducing the propensity of the film to experience edge drying.

For GARField NMR measurements the films were cast onto Menzel Gläser No.0 $18 \mathrm{~mm} \times 18$ $\mathrm{mm}$ borosilicate glass cover slips using a pipette to measure out the dispersion and the pipette tip to spread it. A laminate coating containing a punched hole was applied to the cover slips to create a container for the film to dry in. In this case a $14 \mathrm{~mm}$ wad punch was used to cut the holes in the laminate coating.

\subsection{Atomic Force Microscopy}

A Digital Instruments Atomic Force Microscope was used together with Veeco Nanoscope software to obtain images in tapping mode. 
Image analysis was performed using the Gwyddion software package [15]. A typical AFM image is shown in Figure 1 and the number of big and small particles is easily counted. From this a volume fraction at the top surface is calculated. Typically five areas on each film were analysed. An alternative is to calculate the percentage of area occupied by each particle type at the top surface. Although in this case the numerical answers vary, the qualitative results are similar.

It was noticed from surface scans that there was some radial variation in the dried films, specifically a reduction in the number of larger particles on the surface towards the edges of the samples. In order to obtain a value that would allow for characterisation of the samples, two methods were used:

- Perform scans on multiple films, ensuring that they are taken at the same location on each film, before analysing each one.

- Perform multiple, typically five, scans at different locations on each film, then take an average value from those.

\subsection{Cryogenic Scanning Electron Microscopy}

Films of blends of particles were partially dried on $1 \mathrm{~cm}$ diameter metal platforms within drying chambers. Part way through the drying process the films were taken from the drying chamber and frozen in liquid nitrogen. The films were then placed onto a cold stage at $-120{ }^{\circ} \mathrm{C}$ within the Cryo-SEM module and fractured using a razor blade. After fracture the sample was sublimated at $-95{ }^{\circ} \mathrm{C}$ to allow the particles to protrude from the fractured surface. This was then sputtered with gold and examined by SEM, using a Hitachi S4500 field emission SEM and a Gatan Alto 2500 cryogenic preparation attachment module with cold storage. 


\subsection{GARField NMR}

Measurements of the spatial distribution of mobile ${ }^{1} \mathrm{H}$ in the PIDA polymer in the direction normal to the substrate in dried bimodal latex films was obtained using magnetic resonance ${ }^{1} \mathrm{H}$ profiling with a GARField magnet. The magnet's design has been described elsewhere [16]. Blends of PIDA latex and an acrylic latex with a larger particle size were cast and dried with known Peclet values. Each dried film was placed in the magnet at a position corresponding to a magnetic field strength of $0.7 \mathrm{~T}$ and a field gradient strength of $17.5 \mathrm{Tm}^{-1}$. The NMR signal was obtained using a quadrature echo sequence [17]: (90x- $\tau-90 \mathrm{y}-\tau$-echo- $\tau$-)n for $\mathrm{n}=32$ echoes and a pulse gap of $\tau=95.0 \mathrm{~s}$. To obtain a profile, the echoes were Fourier-transformed and then summed, thus giving an NMR signal intensity profile as a function of vertical position. The NMR intensity is proportional to the density of mobile ${ }^{1} \mathrm{H}$ and was therefore used to determine the distribution of the PIDA particles. The $\mathrm{T}_{2}$ relaxation time is proportional to the mobility of molecules containing ${ }^{1} \mathrm{H}[17,18]$. In these experiments, the glass transition temperature of the acrylic particles was near to the temperature of the GARField measurement (ca. $25^{\circ} \mathrm{C}$ ). Consequently, the molecular mobility - and hence the $\mathrm{T}_{2}$ relaxation time - was relatively low. Hence, no signal was obtained from the standard acrylic particles, however a signal is obtained from the PIDA, which has a higher $\mathrm{T}_{2}$ relaxation time. The pixel resolution achieved in these experiments was about $10 \mu \mathrm{m}$. To correct for the sensitivity decline over the film thickness, profile intensities were normalized by an elastomer standard.

\subsubsection{Analysing images}

The GARField scan gave a profile of just the small, soft particles. A good measure of the stratification is the skewness of this distribution, which is a statistical analysis of the lean of the profile [19]. The calculation of the skewness was carried out only on signal intensities that were higher 
than the noise level on either side of the profile. The skewness, $S_{k}$, is defined as

$$
S_{k}=\frac{m_{3} / m_{0}}{\left(m_{2} / m_{0}\right)^{3 / 2}}
$$

where $m_{i}$ is the $i^{\text {th }}$ moment of the signal intensity. For a distribution in the z-axis of $p$ data points of signal intensity $\varphi$, this is given by

$$
m_{i}=\sum_{j=1}^{j=p} z_{j}^{i} \varphi_{j} \Delta z .
$$

where $z_{j}$ is the vertical position of the intensity and $\Delta z$ is the bin size used to discretise the data. The point on the vertical-axis from which the moments are calculated was set so that the 1st moment is zero. This ensures that a positive skewness value describes a distribution with the long tail to the right hand side, whereas a negative value indicates a long tail to the left hand side. Examples of actual measured profiles with (a) zero, (b) positive and (c) negative skewness are shown in Figure 2. Because the signal obtained from GARField NMR is from the soft particles, a positive skewness value indicates an excess of the soft particles near to the substrate. A negative value indicates an excess of soft particles near to the film/air interface.

\section{Results and Discussion}

\subsection{Particle properties}

Using AFM, it was noticed that the particles were somewhat polydisperse, as shown in Figure 1. All the particles were found to be negatively charged and stable in up to $100 \mathrm{mM}$ sodium chloride solutions. It was noted however that particle mixtures would aggregate slowly over a period of days. This is however longer than any of the drying experiments reported here. 


\subsection{Effect of various experimental parameters}

In this section we show the effect of various experimental parameters. The results from AFM and GARField imaging is compared with the theoretical predictions from the diffusional model, which is described in the previous paper by Trueman et al. [10]. Throughout the results section subscripts 1 and 2 are used to represent the two particle types and the subscript 2 refers to the larger particles with the larger Peclet number.

\subsubsection{Changing the geometric mean Peclet number}

Figure 3 displays example AFM images, obtained from films containing two different particle sizes $(130$ and $380 \mathrm{~nm})$ dried over a range of evaporation rates so as to vary $\sqrt{P e_{1} P e_{2}}$. Each of these surface scans were taken from the centre of the film. It can be seen qualitatively that the image of the film dried with $\sqrt{P e_{1} P e_{2}}=1$ contains more large particles than the others. These and other images were analysed to obtain particle volume fractions at the top surface. Figure 4 shows the data obtained, with the films always being scanned at their center. It can be seen that a maximum in the volume fraction of large particles at the surface occurs around a geometric mean Peclet number of unity. Experimentally, these results show that there is maximum accumulation of large particles at the top surface when the evaporation rate is intermediate. If the evaporation rate is too fast or two slow, then the geometric mean Peclet number is too high or low, respectively.

Figure 5 displays the skewness values obtained from GARField NMR profiling of films dried at varying evaporation rates, which are displayed on the graph as the geometric mean of the Peclet numbers of the two components. For the sample containing $380 \mathrm{~nm}$ acrylic particles $\left(P e_{2} / P e_{1}=\right.$ 2.21 ) it can be seen that although no stratification is observed at higher Peclet numbers, when the evaporation rate is reduced, there are more small particles nearer to the substrate, as expected from the model. For the sample containing $600 \mathrm{~nm}$ acrylic particles $\left(P e_{2} / P e_{1}=3.15\right)$, on the other hand, as the evaporation rate is reduced there are more of the larger particles near to the substrate. 
Theoretical results from the diffusional model predict an accumulation of large, particles near the substrate when the geometric mean Peclet number is unity. The AFM results seem to conform to this prediction, although with a lot of noise. The GARField results seem too noisy to make any definitive prediction. The results for the Peclet ratio of 3.15 seem to suggest an accumulation of small particles at the top surface with the slowest evaporation rates. The most likely explanation for this is an onset of colloidal instability during the slowest drying experiments, resulting in flocs of the large particles and sedimentation, leaving at excess of small, PIDA particles at the top surface.

\subsubsection{Changing the ratio of initial particle volume fractions $\phi_{1_{0}} /\left(\phi_{1_{0}}+\phi_{2_{0}}\right)$}

In the next set of experiments, $P e_{1} / P e_{2}$ and $\sqrt{P e_{1} P e_{2}}$ was fixed, and the initial volume fraction of the two components was varied. The volume fraction ratio of the two components was changed, while ensuring a constant total volume fraction. We use $\phi$ to refer to the volume fractions and the subscripts to distinguish between the different components. The further subscript 0 refers to the initial concentration at time $=0$. AFM images of four different volume fraction ratios can be seen in Figure 6. Surface volume fractions of these films are shown in Figure 7. To facilitate a comparison between samples, the vertical axis displays the percentage increase of the volume fraction of the large particles compared to that which would be expected had the film dried completely uniformly. The trend shows that the stratification increases as the ratio between initial volume fractions of the small and large particles in the sample increases. For the sample with $90 \%$ of the starting volume fraction being small particles there are very few large particles seen on the surface. This magnifies the error in the measurement for this point, since one additional large particle will have a considerable effect of the percentage increase above a uniform value.

When measuring films with GARField NMR it was not possible to obtain a significant signal above the noise value from films with less than $50 \%$ small particles. The skewness of the profiles obtained for different volume fraction ratios of the two components are displayed in Figure 8. For both particle blends the skewness increases as $\phi_{1_{0}} /\left(\phi_{1_{0}}+\phi_{2_{0}}\right)$ increases. This indicates an 
increase in the amount of large particles at the top of the film.

The modelling work [10] predicted that as $\phi_{10} /\left(\phi_{10}+\phi_{20}\right)$ increased, the percentage increase of the large particles at the surface would increase. This was also seen with both experimental techniques. It is noticeable that in these experiments the value of $\sqrt{P e_{1} P e_{2}}$ was such that the sedimentation, expected at the very slowest evaporation rates, was not observed.

\subsubsection{Changing the initial volume fraction $\phi_{1_{0}}+\phi_{2_{0}}$}

In the next set of experiments, the total initial volume fraction of particles was changed. That is, the ratio of the volume fractions of the two components was fixed while the blends were diluted by varying amounts. Figure 9 shows the volume fraction of large particles at the surface increasing as the total initial volume fraction of particles decreases. These samples were dried with constant $P e_{2} / P e_{1}, \phi_{10} / \phi_{20}$ and $\sqrt{P e_{1} P e_{2}}$.

Figure 10 displays the change in skewness as the total initial volume fraction of the dried samples is varied. The trend is the same for both particle blends - the skewness increases as the total initial volume fraction decreases.

The observation with each technique was that as the total initial volume fraction was decreased, the volume fraction of large particles near the top surface of the films increased. This behaviour was as predicted theoretically [10]. The lower initial volume fractions allow more time for a diffusional stratification to occur.

\subsubsection{Changing $P e_{2} / P e_{1}$ via the size ratio}

In a final set of experiments, the evaporation rate and volume fractions were fixed, but the size ratio of the two components was varied, as a means of adjusting $P e_{2} / P e_{1}$. Images produced from the surface topography of films dried with different size ratios of the particles are displayed in 
Figure 11. For a size ratio of $R_{2} / R_{1}=1.73$ it was not possible to sufficiently distinguish the two particles from one another. For the largest two size ratios very few large particles were seen on the surface. It is also difficult to tell whether the protrusions from the surface in these images are large particles or collections of small particles because of the surface inhomogeneities in the surfaces of the $500 \mathrm{~nm}$ and $600 \mathrm{~nm}$ particles.

The GARField NMR results can be seen in Figure 12. It is evident that the skewness decreases as the size ratio between the two components increases. This is indicative of more small particles towards the top surface as the size ratio increases. This is opposite to the theoretical prediction of Trueman et al. [10]. The most likely explanation for the discrepancy is again an increase in dispersion flocculation with larger size disparities. Depletion interactions are stronger with larger size disparities and the increase in floc size would then lead to an increased sedimentation of the larger particles and the resulting increase in small particles towards the top surface.

\subsection{Cryo-SEM and potential issues with AFM imaging}

AFM is a technique that, unless cross-sections of the film are carefully cut, scans only the top surface of the dried films. In some situations this is the main area of interest, however for a more complete characterisation it can be useful to obtain data from regions throughout the depth of the

film. One technique that can be used to obtain such data is Cryo-SEM [1]. Figure 13 shows an image taken of a film containing particles of two differing sizes. It is clear from these images that the surface composition is different from that immediately beneath, with there being a higher volume of small particles at the surface than immediately below. Whilst surface techniques such as AFM and SEM are simple to perform, the reliance on the top surface is a major drawback. Hence whilst trends in the particle stratification are obtainable, any quantitative information should not be relied on from the AFM data. As will be shown [12] an attraction for one particle type to the top surface can lead to complete enrichment of the surface with just those particles, with the layer immediately below being the value that would have occurred without the surface attraction. 
Another issue with the AFM method is that the particle counting technique was an automated one. To ensure that each sample was measured in a consistent manner a cut-off size was chosen which would discern between large and small particles. This was applied equally to every image taken. It is clear in some of the images that there are some particles for which it is difficult to ascertain whether they are small or large, generally because they are obscured by others. The automated technique usually counts these as small particles, hence the true number of large particles may be underestimated, although this will be a systematic error.

\subsubsection{GARField NMR}

The main advantage of GARField NMR is that it produces vertical profiles through the film. It is also a direct measurement of the soft particles. The resolution of profiles obtained using GARField NMR is about $10 \mu \mathrm{m}$ and whilst this is sufficient to observe the presence of a gradient in composition throughout the film, it is not high enough to observe any layers of just a few particles. A significant disadvantage of using GARField NMR is that the samples are restricted to a diameter of $14 \mathrm{~mm}$ when the laminate coating is used. This means that any edge drying effects will be more significant than with the larger radii film in samples prepared for AFM. This manifested itself visually, as the level of turbidity at the center and edges differed. This type of behaviour from drying films was also noticed by Yang et al. [20].

\subsection{Other sources of particle transport}

The experimental results indicate the presence of a diffusional stratification. It is also evident that these films are far more complex than the simplified diffusional model would hope. There are a number of other flow mechanisms that need to be considered:

The small particles may have a chemical attraction to the top surface, perhaps due to the surface chemistry of the particles, which would lead to them becoming enriched at the top surface, yet 
the rest of the film would dry as normal. Evidence for this type of stratification comes from the Cryo-SEM image Figure 13. The interactions between particles and attraction for one particle type to an interface is easily included in the existing model and is the subject of a future paper [12].

Colloidal stability has been a major issue in this work. Aggregation of the larger particles, followed by sedimentation, will lead to the larger particles being found predominantly near the substrate. In a bidisperse system flocculation is likely to occur due to depletion interactions [11]. We ensured visual colloidal stability for up to 10 days with our particle mixtures. However formation of small weak aggregates would promote sedimentation and may explain why we consistently observe an excess of large particles towards the substrate.

Convection currents can form within a drying film due to temperature differences between different locations. Any such currents would be likely to drag the small particles more than the large ones. Care was taken to ensure the films dried in an as undisturbed form as possible, to reduce the chance of the presence of any convection currents, however their presence is certainly possible.

Any surfactant in the film will inevitably migrate to the film-air interface. This will then lead to Marangoni flows which will dominate any diffusional stratification. In these experiments the latexes were extensively dialysed to try to remove any surfactants although their presence is always possible.

It was demonstrated by Nikiforow et al. [21] that the charge on the surface of colloids can cause a mixture of charged and non-charged particles to segregate based on differing diffusion rates. The zeta potentials of the particles used in this study were found to be similar. It seems likely that if the surface charge magnitudes were very different then this type of stratification would occur, however it is not certain whether the small differences in the surface charges of the particles used in this present work would demonstrate the same phenomena.

Lateral drying was a perennial difficulty. It was minimised by use of the laminate surround, but not 
completely removed. This means that any experimental measurements are subject to their lateral location and, especially for AFM measurements, many such measurements need to be averaged. This introduces an inevitable error into the experiments.

\section{Conclusions}

We have demonstrated how the particle size ratio can be used to set a ratio of Peclet numbers for the components in a colloidal blend. The evaporation rate can then be adjusted to vary the geometric mean Peclet number such that it is near unity.

We have used two different experimental techniques to examine stratification in drying latex films. AFM cannot distinguish between concentration profiles in the depth of a film and segregation of particles at a surface. The cryo-SEM analysis has shown definitive evidence for the latter. GARField NMR provides concentration profiles through the film, and the profile skewness is a useful measure of stratification.

Irrespective of the technique used, there is clear evidence for stratification in drying films. We found that greater stratification is observed at lower initial volume fractions and with a low concentration of the segregating component. There was some evidence from AFM for enhanced stratification at a geometric mean Peclet number of unity, although the GARField data was inconclusive in this respect.

These experimental results, when combined with the results from the modelling work presented by Trueman et al. [10], demonstrate that with careful control of the drying environment, the presence of different sized particles can be used to manipulate the film morphology. In order to create a system in which stratification is most likely to be encouraged, the following conditions should therefore be achieved:

- The Peclet numbers of the two components should lie either side of unity. 
- The total initial volume fraction of the particles should be low enough to enable the particles to diffuse.

- The size difference should be great enough to have a large difference in diffusivities between the two components. The particles must not phase separate or aggregate under depletion flocculation when blended, however, which restricts the maximum size ratio between the two components.

\section{Acknowledgements}

The authors are grateful to Prof. Diethelm Johannsmann (Clausthal University of Technology, Germany) and Dr. Stuart Clarke (University of Cambridge) for helpful discussions and to Michael A. Rabjohns and Professor Peter Lovell (University of Manchester) for the PIDA sample. RET was helped with GARField experiments by Andre Utgenannt, Carolina de las Heras and Radek Kowalczyk. RET was supported by a CASE award from ICI/AkzoNobel and EPSRC. 


\section{References}

[1] Luo, H.; Scriven, L. E.; Francis, L. F. Journal of Colloid and Interface Science 2007, 316, 500-509.

[2] Luo, H.; Cardinal, C. M.; Scriven, L. E.; Francis, L. F. Langmuir 2008, 24, 5552-5561.

[3] Keddie, J. L.; Routh, A. F. Fundamentals of latex film formation; Processes and Properties; Springer, 2010.

[4] Routh, A. F.; Russel, W. B. AIChE Journal 1998, 44, 2088-2098.

[5] Deegan, R. D.; Bakajin, O.; Dupont, T. F.; Huber, G.; Nagel, S. R.; Witten, T. A. Nature 1997, 389, $827-829$.

[6] Goehring, L.; Clegg, W. J.; Routh, A. F. Langmuir 2010, 26, 9269-9275.

[7] Goehring, L.; Clegg, W. J.; Routh, A. F. Soft Matter 2011, 7, 7984-7987.

[8] Routh, A. F.; Zimmerman, W. B. Chemical Engineering Science 2004, 59, 2961-2968.

[9] Ekanayake, P.; McDonald, P. J.; Keddie, J. L. European Physical Journal - Special Topics 2009, 166, $21-27$.

[10] Trueman, R. E.; Lago Domingues, E.; Emmett, S. N.; Murray, M.; Routh, A. F. submitted to Langmuir 2011,

[11] Russel, W. B.; Saville, D. A.; Schowalter, W. R. Colloidal Dispersions; Cambridge University Press, 1991.

[12] Atmuri, A.; Bhatia, A. F., S. R. Routh submitted to Langmuir 2011,

[13] Carra, S.; Pinoci, D. Macromolecular Symposia 2002, 187, 585-596.

[14] Gorce, J. P.; Bovey, D.; McDonald, P. J.; Palasz, P.; Taylor, D.; Keddie, J. L. European Physical Journal E 2002, 8, 421-429.

[15] Klapetek, P.; Neas, D.; Anderson, C. Gwyddion user guide, http://gwyddion.net/. 2004-2009.

[16] Glover, P. M.; Aptaker, P. S.; Bowler, J. R.; Ciampi, E.; McDonald, P. J. Journal of Magnetic Resonance 1999, 139, 90 - 97. 
[17] McDonald, P. J.; Newling, B. Reports on Progress in Physics 1998, 61, 1441.

[18] Wang, T.; de las Heras Alarcon, C.; Goikoetxea, M.; Beristain, I.; Paulis, M.; Barandiaran, M. J.; Asua, J. M.; Keddie, J. L. Langmuir 2010, 26, 323-333.

[19] König, A. M.; Weerakkody, T. G.; Keddie, J. L.; Johannsmann, D. Langmuir 2008, 24, 7580-7589.

[20] Yang, Z. Z.; Wang, L. J.; Liu, Z. P.; Zhao, D. L. Journal of Applied Polymer Science 2001, 80, $1835-1840$.

[21] Nikiforow, I.; Adams, J.; König, A. M.; Langhoff, A.; Pohl, K.; Turshatov, A.; Johannsmann, D. Langmuir 2010, 26, 13162-13167. 


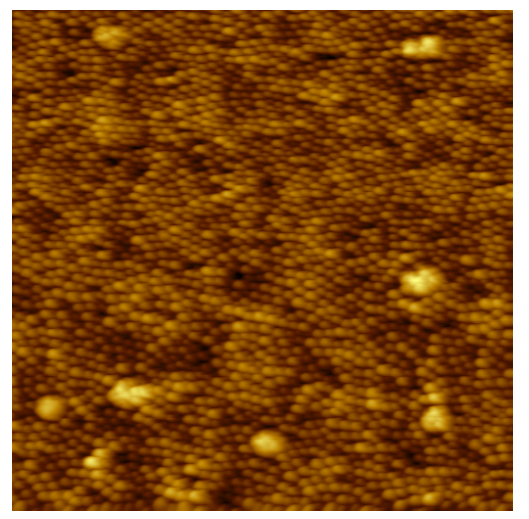

Figure 1: Topographic image of surface of a dried film of the high $T_{g}$ acrylic latex 2, as obtained by AFM. Image dimensions: $5 \mu \mathrm{m} \times 5 \mu \mathrm{m}$.

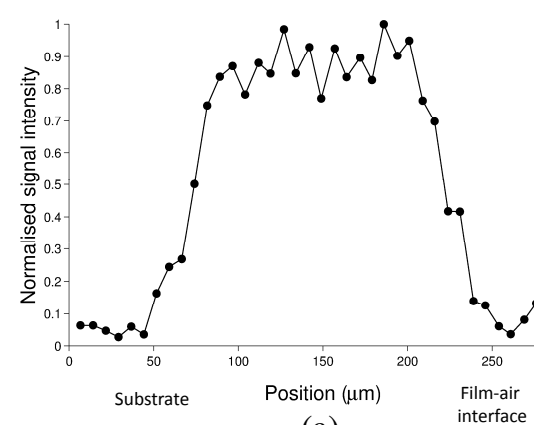

(a)

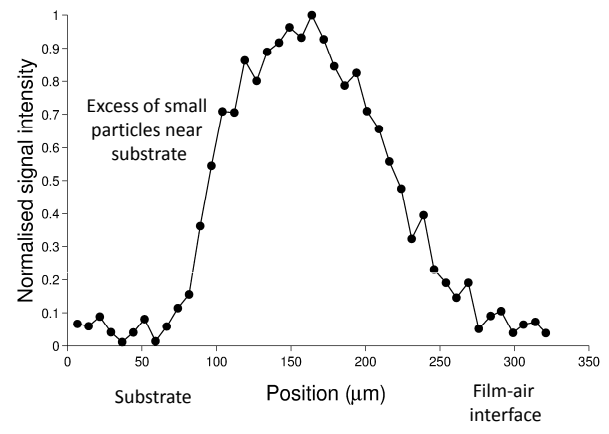

(b)

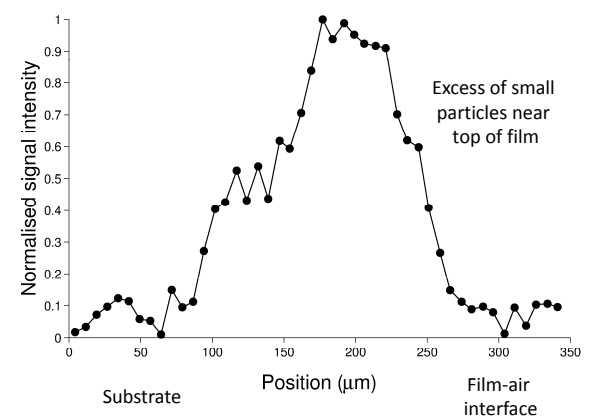

(c)

Figure 2: GARField distributions displaying (a) zero (b) positive and (c) negative skewness. 


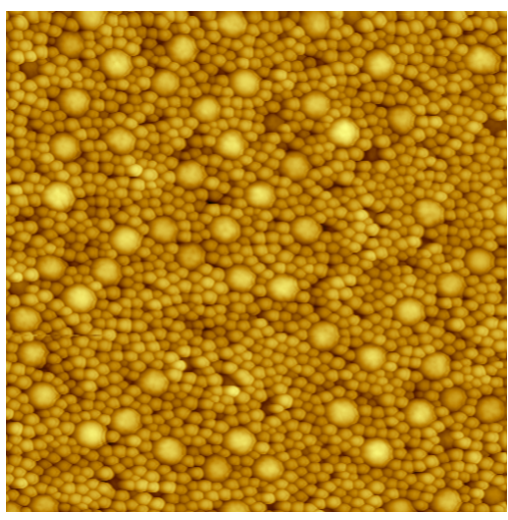

(a) $\sqrt{P e_{1} P e_{2}}=0.2$

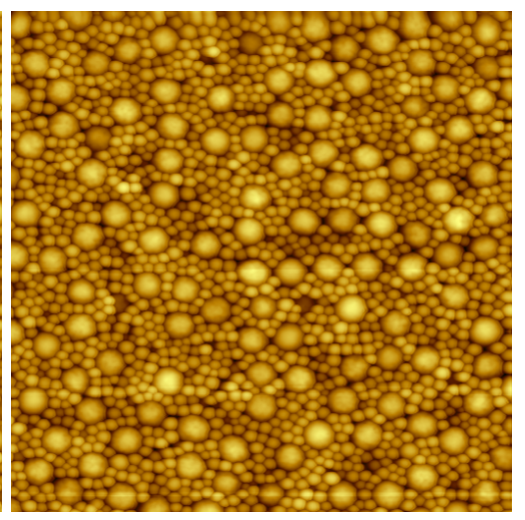

(b) $\sqrt{P e_{1} P e_{2}}=1$

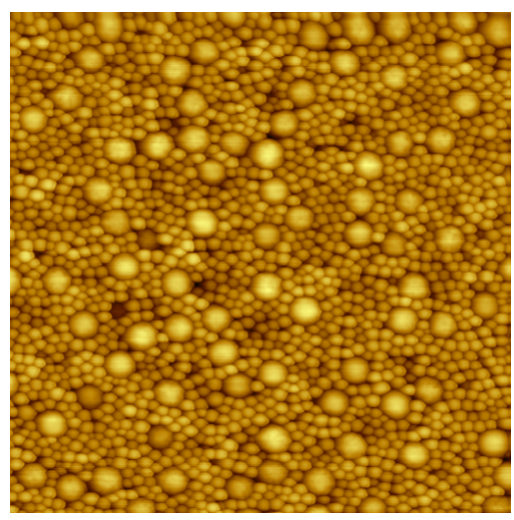

(c) $\sqrt{P e_{1} P e_{2}}=3$

Figure 3: Topographic AFM images of film surfaces obtained with varying geometric mean Peclet numbers, $\sqrt{P e_{1} P e_{2}}$. Experimental conditions are $\phi_{1_{0}}=0.1, \phi_{2_{0}}=0.1, P e_{2} / P e_{1}=2.94$. The magnitude of $\sqrt{P e_{1} P e_{2}}$ is increased by increasing the evaporation rate. Image dimensions: $5 \mu \mathrm{m}$ $\times 5 \mu \mathrm{m}$. 


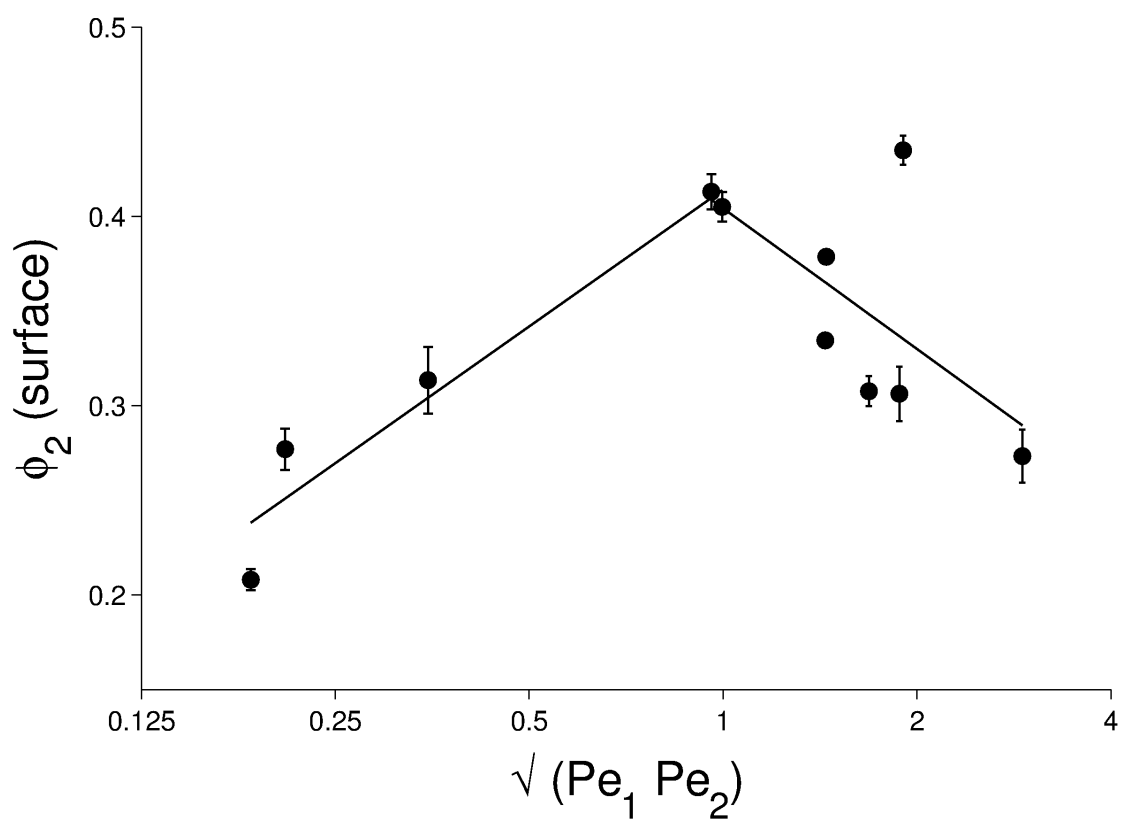

Figure 4: Volume fraction of the large particles at the surface of films dried with varying geometric mean Peclet number. The experimental conditions are $\phi_{10}=0.1, \phi_{20}=0.1$ and $P e_{2} / P e_{1}=2.94$. Lines added as a guide to the eye.

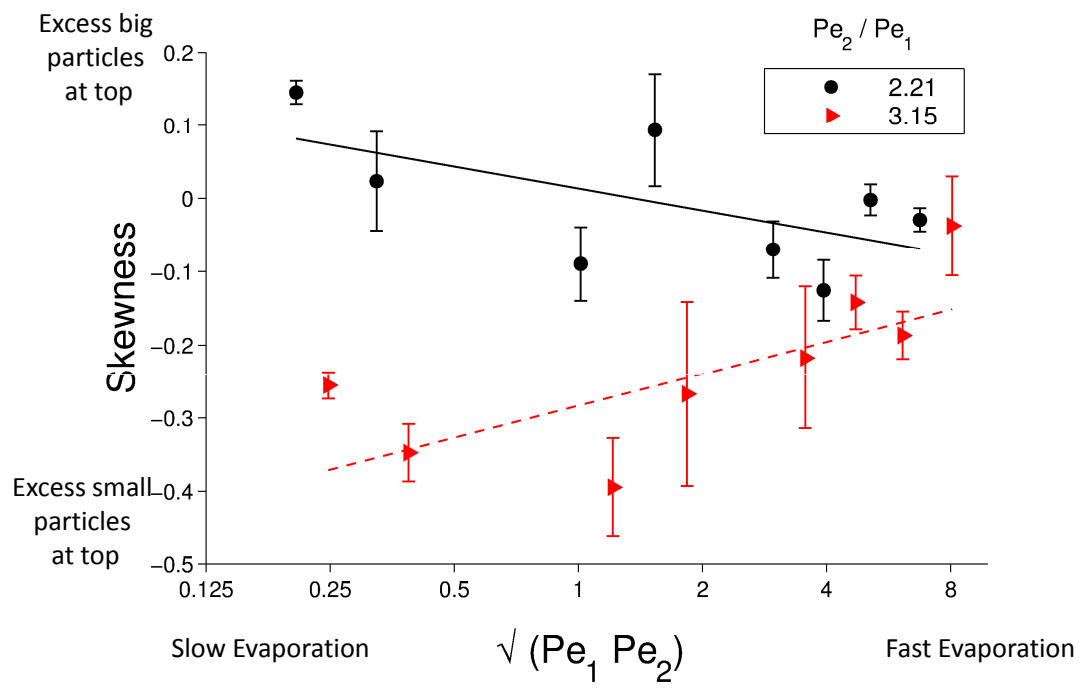

Figure 5: Skewness of profiles obtained from GARField NMR through dried blends of particles with varying geometric mean Peclet number, $\sqrt{P e_{1} P e_{2}}$. Experimental conditions are $\phi_{10}=0.125$ and $\phi_{20}=0.125$. Lines are added as a guide to the eye. 


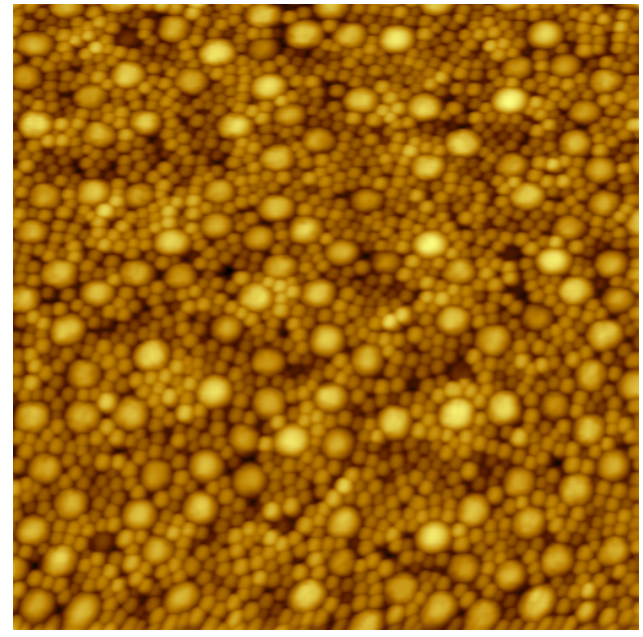

(a) $\phi_{10} /\left(\phi_{10}+\phi_{20}\right)=0.25$

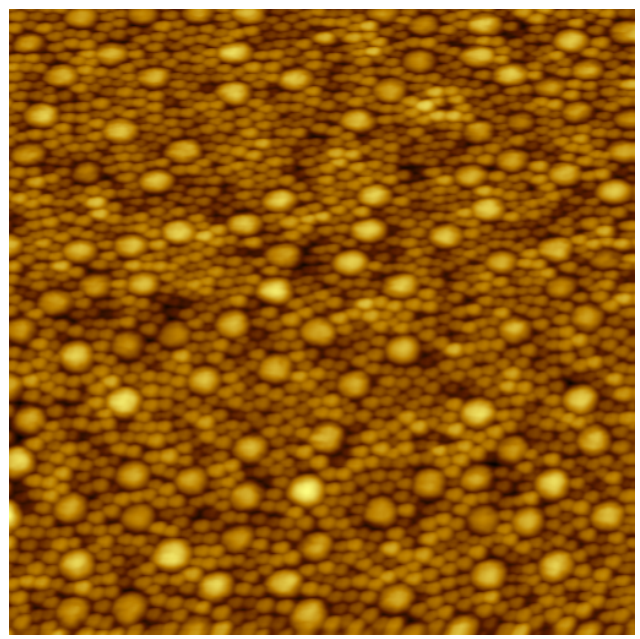

(c) $\phi_{10} /\left(\phi_{10}+\phi_{20}\right)=0.75$

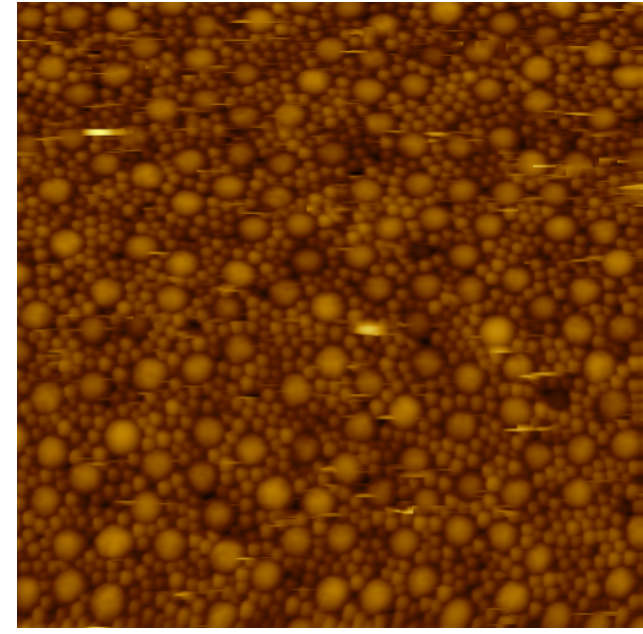

(b) $\phi_{10} /\left(\phi_{10}+\phi_{20}\right)=0.5$

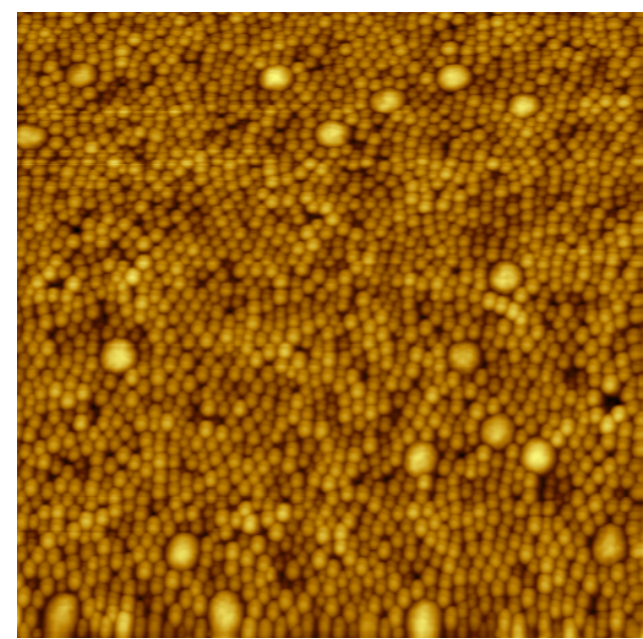

(d) $\phi_{10} /\left(\phi_{10}+\phi_{20}\right)=0.9$

Figure 6: Topographic AFM data of films dried with differing initial volume fraction ratios between the two components. Experimental conditions were $\sqrt{P e_{1} P e_{2}}=1.56, \phi_{10}+\phi_{20}=0.25$ and $P e_{2} / P e_{1}=2.94$. Image dimensions: $5 \mu \mathrm{m} \times 5 \mu \mathrm{m}$. 


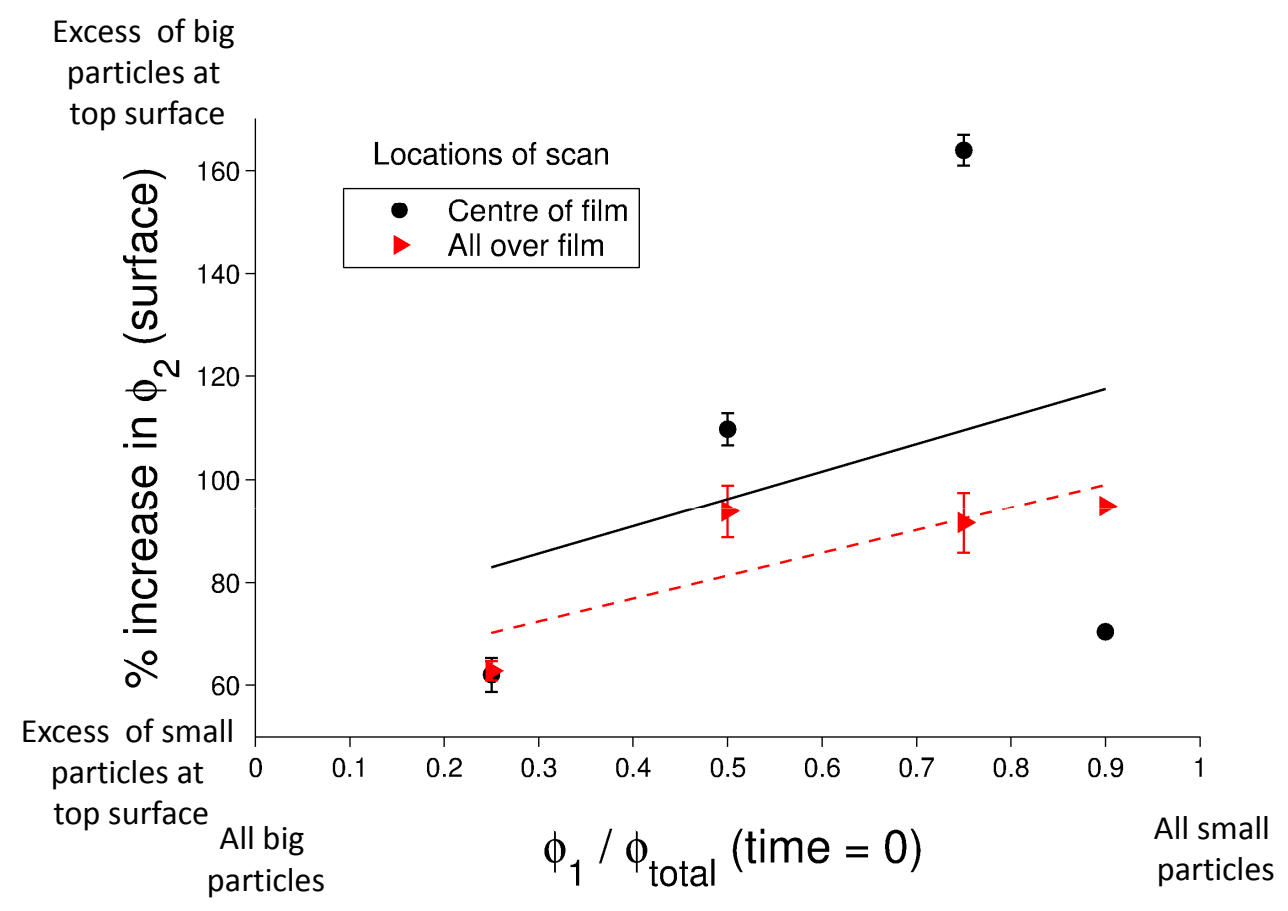

Figure 7: Percentage increase in volume fraction of large particles at the surface of films with varying $\phi_{10} /\left(\phi_{1_{0}}+\phi_{2_{0}}\right)$. The experimental conditions were $P e_{2} / P e_{1}=2.94, \sqrt{P e_{1} P e_{2}}=1.56$, $\phi_{10}+\phi_{20}=0.25$. Lines are added as a guide to the eye.

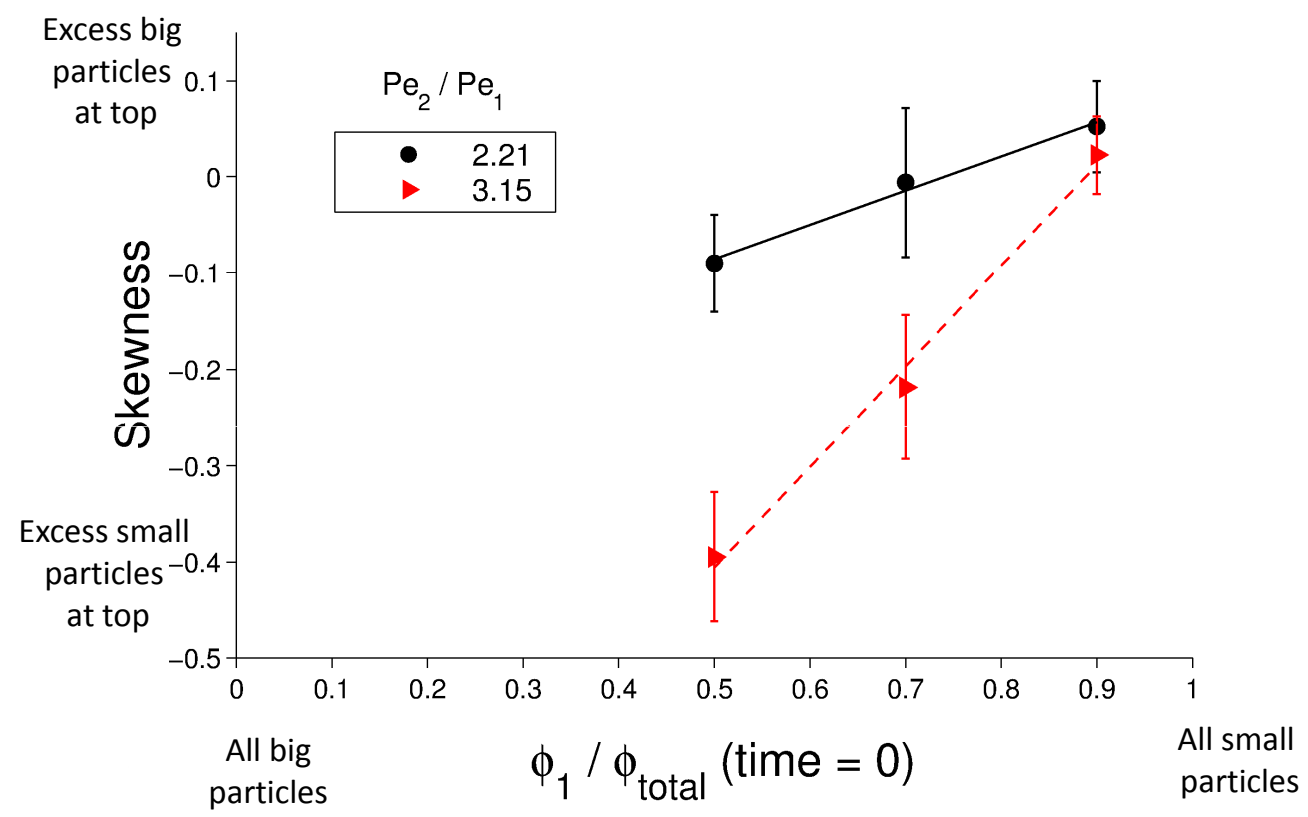

Figure 8: Skewness of profiles obtained from GARField NMR through dried blends of acrylic and PIDA particles with varying ratio of initial volume fraction, $\phi_{10} /\left(\phi_{10}+\phi_{20}\right)$. The experimental conditions were $P e_{1}=0.71$ and $\phi_{10}+\phi_{20}=0.25$. Lines are added as a guide to the eye. 


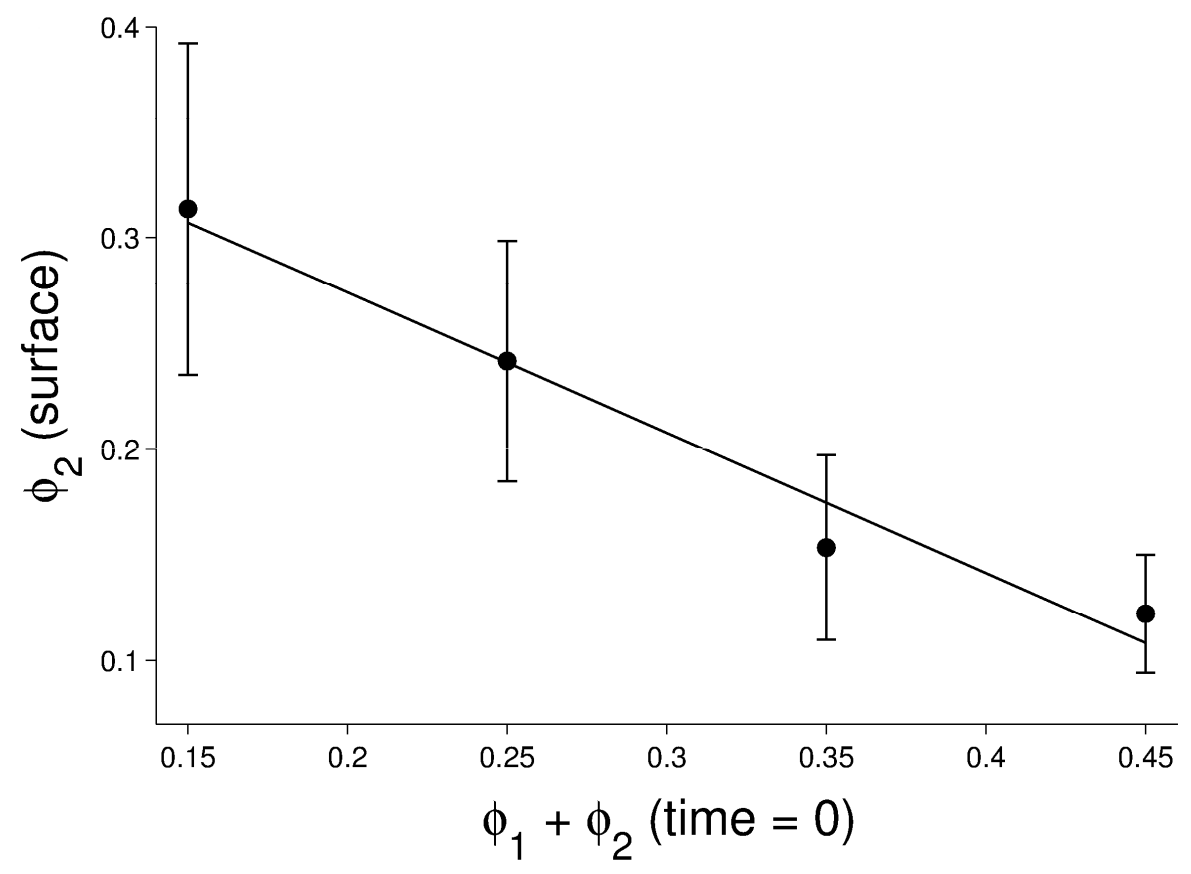

Figure 9: Volume fraction of large particles at the surface of films dried with changing initial volume fraction, $\phi_{10}+\phi_{20}$. Experimental conditions were $P e_{2} / P e_{1}=2.94, \phi_{10} / \phi_{20}=1$ and $\sqrt{P e_{1} P e_{2}}=1.27$. Line is added as a guide to the eye.

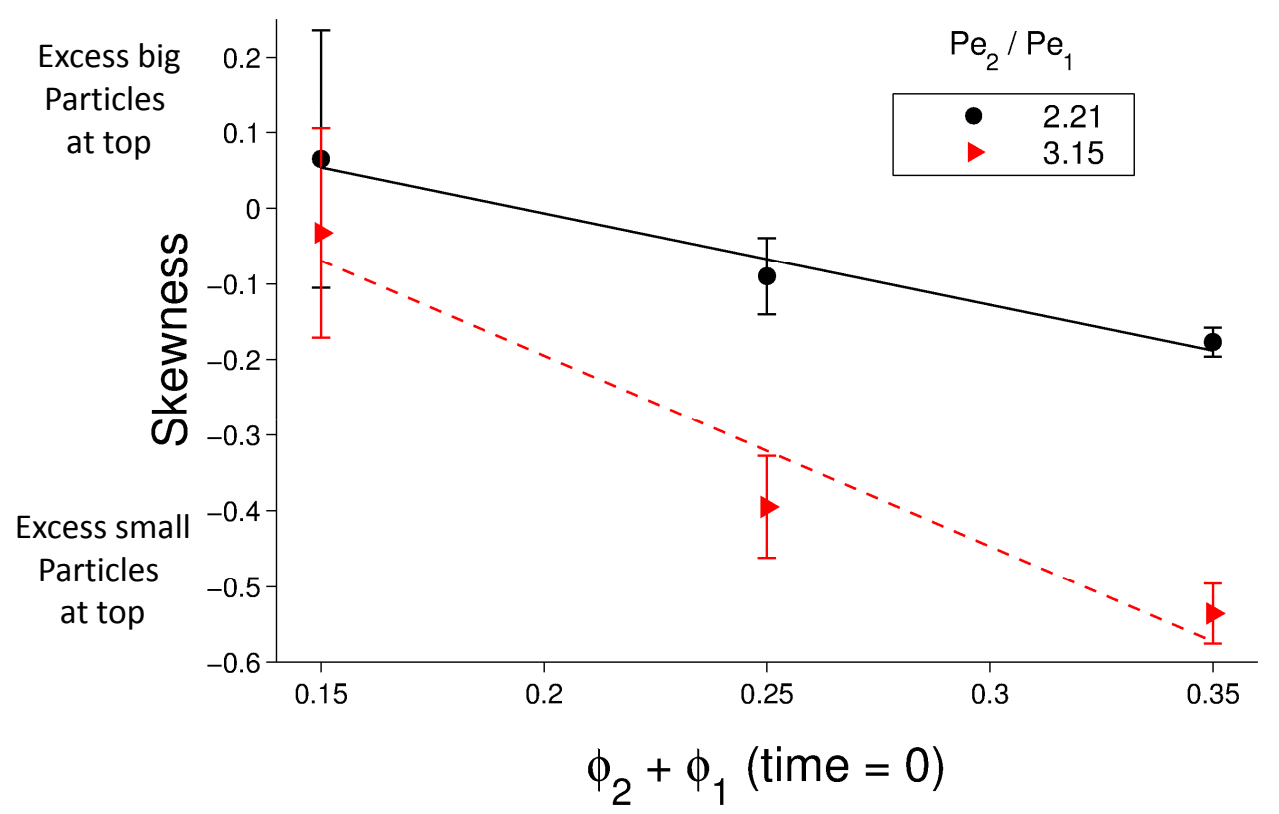

Figure 10: Skewness of profiles obtained from GARField NMR through dried blends of acrylic and PIDA particles with varying initial volume fraction, $\phi_{1_{0}}+\phi_{2_{0}}$. Experimental conditions were $\phi_{10} / \phi_{20}=1$ and $P e_{1}=0.65$. Lines are added as a guide to the eye. 


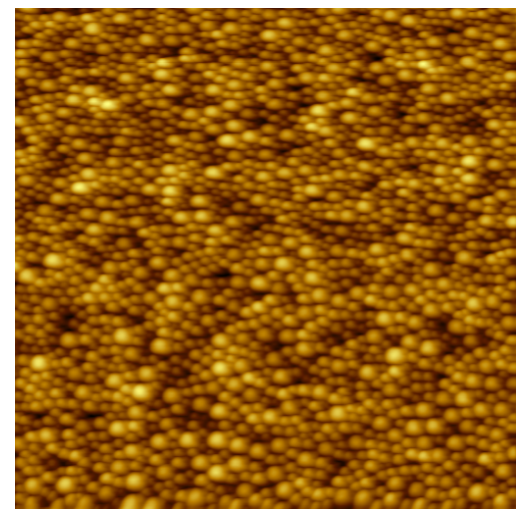

(a) $P e_{2} / P e_{1}=1.73$

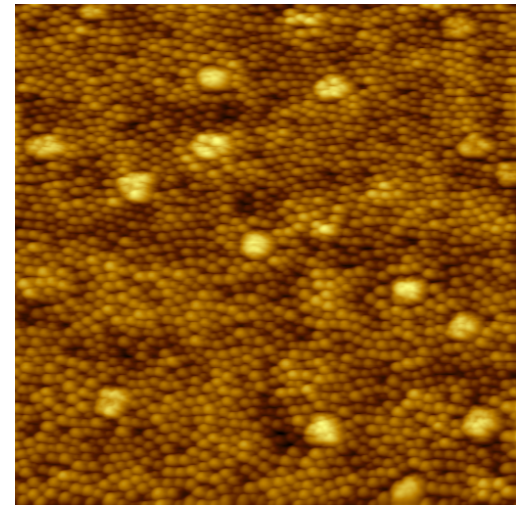

(c) $P e_{2} / P e_{1}=4.19$

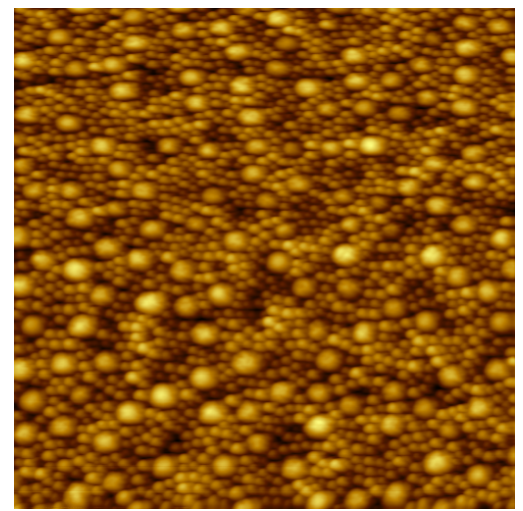

(b) $P e_{2} / P e_{1}=2.94$

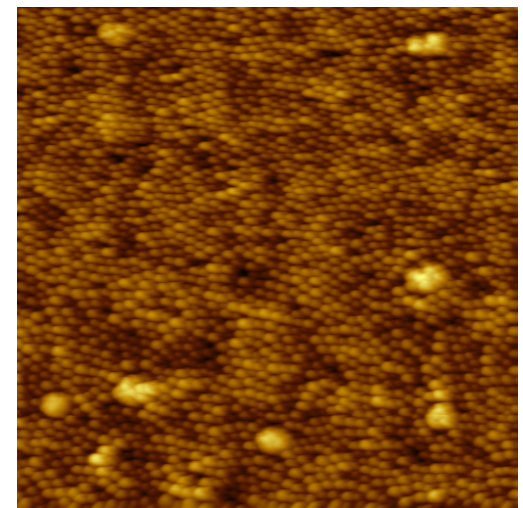

(d) $P e_{2} / P e_{1}=4.97$

Figure 11: Topographic AFM images, of acrylic blend films, at different particle size ratios, $R_{2} / R_{1}$ leading to different $P e_{2} / P e_{1}$ ratios. The experimental conditions were $\phi_{1_{0}}=0.125$, $\phi_{20}=0.125$ and $P e_{1}=0.91$ Image dimensions: $5 \mu \mathrm{m} \times 5 \mu \mathrm{m}$.

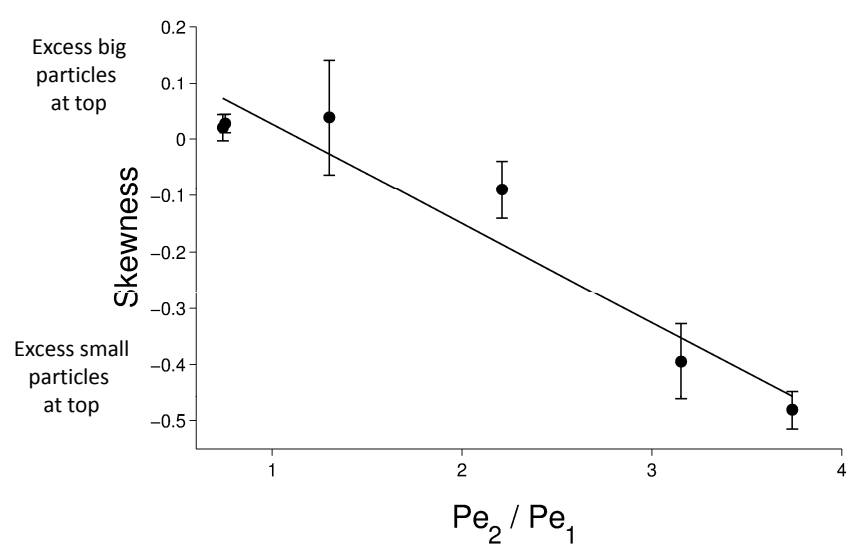

Figure 12: Skewness of profiles obtained from GARField NMR through dried blends of particles with varying size ratio between acrylic and PIDA particles, $P e_{2} / P e_{1}$. Experimental conditions were $\phi_{10}=0.125, \phi_{20}=0.125$ and $P e_{1}=0.65$. Line is added as a guide to the eye. 


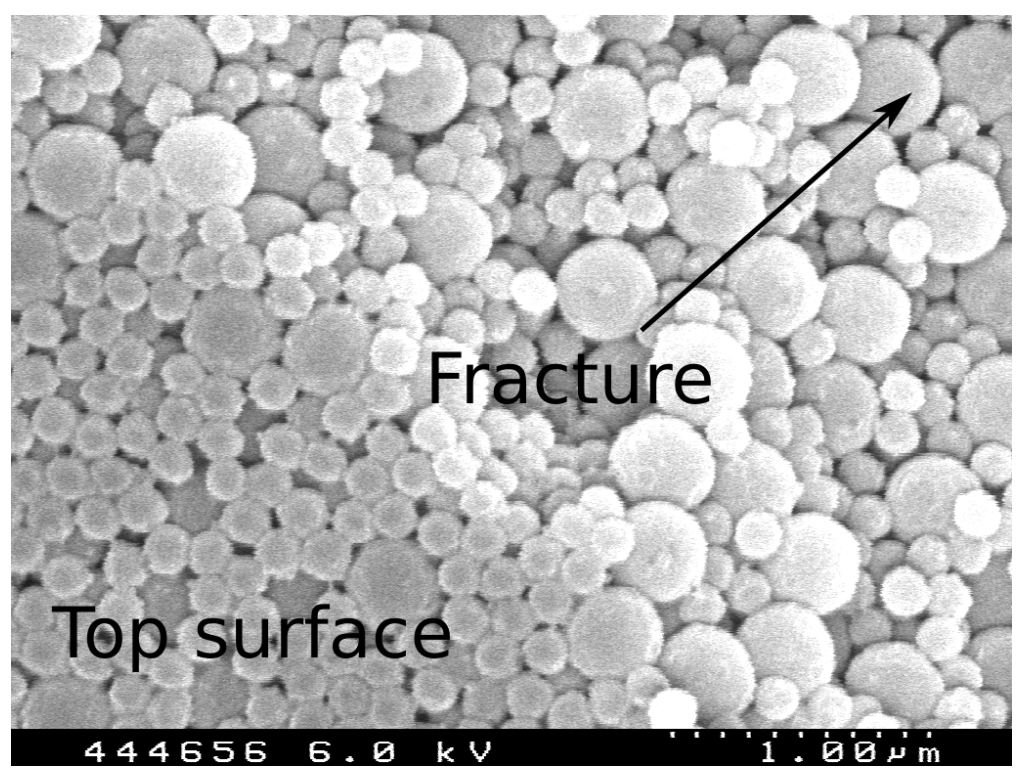

Figure 13: Cryo-SEM image of blend of blend of Acrylic $1(130 \mathrm{~nm})$ and Acrylic $3(380 \mathrm{~nm})$ particles, looking from the surface into a fracture through the film. 
For Table of Contents Page only

Auto-stratification in drying colloidal dispersions: Experimental investigations by Trueman, Lago Domingues, Emmett, Murray, Keddie and Routh

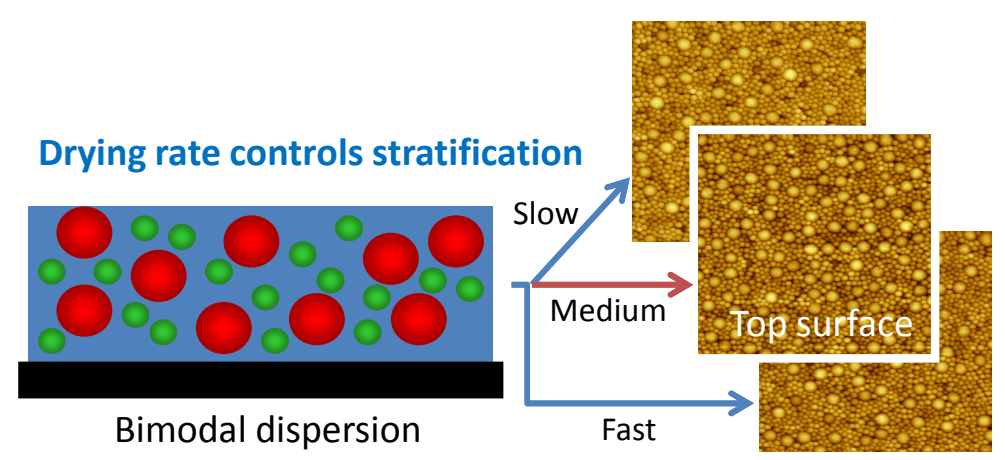

